\title{
Non-invasive detection of nasopharyngeal carcinoma using saliva surface-enhanced Raman spectroscopy
}

\author{
SUFANG QIU ${ }^{1 *}$, YUANJI XU $^{1 *}$, LINGLING HUANG ${ }^{1}$, WEI ZHENG ${ }^{2}$, CHAOBIN HUANG $^{2}$, SHAOHUA HUANG $^{3}$, \\ JINYONG LIN ${ }^{3}$, DUO LIN ${ }^{4}$, SHANGYUAN FENG ${ }^{3}$, RONG CHEN $^{3 * *}$ and JIANJI PAN ${ }^{1,2 * *}$ \\ ${ }^{1}$ The Shengli Clinical Medical College of Fujian Medical University, Fuzhou, Fujian 350001; \\ ${ }^{2}$ Department of Radiation Oncology, Fujian Provincial Cancer Hospital, Fuzhou, Fujian 350014; \\ ${ }^{3}$ Key Laboratory of OptoElectronic Science and Technology for Medicine, Ministry of Education, Fujian Normal University, \\ Fuzhou, Fujian 350007; ${ }^{4}$ College of Integrated Traditional Chinese and Western Medicine, \\ Fujian University of Traditional Chinese Medicine, Fuzhou, Fujian 350122, P.R. China
}

Received December 16, 2014; Accepted September 28, 2015

DOI: $10.3892 / \mathrm{ol} .2015 .3969$

\begin{abstract}
The present study evaluated the use of saliva surface-enhanced Raman spectroscopy (SERS) for the detection of non-invasive nasopharyngeal carcinoma (NPC). SERS measurements were taken from 62 saliva samples, of which 32 were from NPC patients and 30 from healthy volunteers. Notable biochemical Raman bands in the SERS spectra were tentatively assigned to various saliva components. The saliva SERS spectra obtained from the NPC patients and the healthy volunteers were also analyzed by multivariate statistical techniques based on principal component analysis and linear discriminant analysis (PCA-LDA). Significant differences were observed between the saliva SERS spectral intensities for NPC patients and healthy volunteers, particularly at 447, 496, 635, 729, 1134, 1270 and $1448 \mathrm{~cm}^{-1}$, which primarily contained signals associated with proteins, nucleic acids, fatty acids, glycogen and collagen. The classification results based on the PCA-LDA method provided a relatively high diagnostic sensitivity of $86.7 \%$, specificity of $81.3 \%$ and diagnostic accuracy of $83.9 \%$ for NPC identification. The results from the present study demonstrate that saliva SERS analysis used in conjunction with PCA-LDA diagnostic algorithms possesses
\end{abstract}

Correspondence to: Mr. Jianji Pan, Department of Radiation Oncology, Fujian Provincial Cancer Hospital, 420 Fuma Road, Fuzhou, Fujian 350014, P.R. China

E-mail: panjianji@aliyun.com

Mr. Rong Chen, Key Laboratory of OptoElectronic Science and Technology for Medicine, Ministry of Education, Fujian Normal University, 32 Shangsan Road, Fuzhou, Fujian 350007, P.R. China

E-mail: chenr@fjnu.edu.cn

"Co-first authors; ${ }^{* *}$ Contributed equally

Key words: SERS, saliva, principal component analysis, nasopharyngeal carcinoma, non-invasive detection a promising clinical application for the non-invasive detection of NPC.

\section{Introduction}

Nasopharyngeal carcinoma (NPC) is considered to be an endemic carcinoma in Southern China, and Fujian has one of the highest incidence rates (1). The 5-year overall survival rate ranges between 84 and $90 \%$ for early-stage NPC patients (2), whereas advanced stages have a poor 5-year overall survival rate of $30.3-73.6 \%$ (3). Therefore, early detection and diagnosis are critical to prevent the advancement of NPC and improve the 5-year overall survival rate. At present, diagnostic methods for NPC include blood tests, flexible fiber-optic nasopharyngoscopy, chest computed tomography (CT), nasopharyngeal and neck magnetic resonance imaging, bone emission CT, body position emission tomography (PET) and pathological biopsy, which are invasive, inconvenient and time-consuming, and the interpretation of results relies on the experience of the physician (4-7). For example, pathological biopsy usually involves chemical staining, and CT or PET examination usually requires. These diagnostic methods may alter the native physiological status of the patient (8). In addition, the diagnostic methods do not achieve an ideal diagnostic sensitivity and specificity. Therefore, obtaining a diagnostic tool for NPC that may overcome the disadvantages observed with other diagnostic methods is imperative.

Raman spectroscopy (RS) is a unique vibrational spectroscopic and non-invasive laser-based analytical technique that aids biochemical component analysis. RS is based on the molecular vibrations that are specific to certain types of biomolecules, including proteins, nucleic acids and lipids (9). In previous years, there has been an increasing focus on the biomedical applications of RS, including the application of RS for oncology diagnosis to detect the differences between normal and malignant tissue in diseases including lung, colonic, bronchial and skin cancers (10-13). However, Raman scattering is a weak process, with 1 out of $10^{6}-10^{8}$ photons becoming inelastically scattered (14). In addition, the strong autofluorescence background caused by optical components 
severely interferes with the detection of a weak Raman signal (15). These complications hinder the clinical application of RS.

With the advent of nanotechnology and its application in medicine, surface-enhanced RS (SERS) has emerged to solve the challenges encountered with RS (16). SERS enhances the Raman signal by $10^{9}-10^{14}$ times, due to the analytes becoming absorbed by or becoming close to a roughened coinage metallic surface or nanoparticles (16). Furthermore, the strong autofluorescence background is greatly reduced, due to the absorption of molecules on the metal nanoparticles $(17,18)$. The advantages of SERS establish the technique as a promising candidate for analyzing biomolecules, including DNA, cells, viruses and bacteria (19-22). In addition, a number of studies have demonstrated successful results concerning SERS application for oncology diagnosis, which have ideal diagnostic sensitivities, specificities and accuracies (23-25). The most common samples taken for SERS diagnosis in oncology are blood, tissue, cells, DNA and RNA. For NPC detection, blood and tissue are the samples most likely to be taken $(18,25)$. However, the obtainment of these human samples during carcinoma diagnosis, treatment and follow-up is invasive and inconvenient. This leads to the consideration of whether there is a more convenient sample for the diagnosis of NPC using SERS.

In cancer patients, the composition and structure of biomolecules in human saliva alter, due to the apoptosis and necrosis of cells (26). In addition, saliva samples may be collected non-invasively, quickly, repeatedly and economically. To the best of our knowledge only 2 studies have outlined the efficiency of saliva SERS diagnosis of oral and lung carcinoma $(27,28)$. However, the studies assessed a limited number of carcinoma samples, consisting of 4 oral carcinoma patients and 21 lung carcinoma patients. Recently, Feng et al combined membrane protein purification with SERS in NPC patient saliva analysis (29), which achieved a diagnostic accuracy of $90.2 \%$, sensitivity of $91.9 \%$ and specificity of $76.7 \%$. However, the present study only analyzed the proteins in saliva, which is only one of the numerous components contained in saliva, leading to a loss of diagnostic information associated with the alteration of biochemical components in NPC patient saliva. Furthermore, the diagnosis or screening of carcinomas requires convenience and haste, whereas membrane protein purification, performed by Feng et al, increased the complexity of the saliva SERS methodology.

The aim of the present study was to explore the implementation of the saliva SERS method to differentiate NPC patients from healthy volunteers. Multivariate statistical techniques, including principal component analysis (PCA) and linear discriminant analysis (LDA), were employed to develop diagnostic algorithms for differentiation. The receiver operating characteristic (ROC) curve was calculated to additionally evaluate the accuracy of the diagnostic algorithm.

\section{Materials and methods}

Preparation of saliva samples and silver (Ag) colloids. Ethical approval was obtained from Fujian Provincial Cancer Hospital (Fuzhou, Fujian, China). The saliva samples of 32 patients with pathologically-confirmed NPC and 30 healthy volunteers were collected at Fujian Provincial Cancer Hospital. Detailed information concerning the study participants is presented in Table I. The saliva samples were collected as follows: Each participant gargled 3 times prior to breakfast; $\sim 1 \mathrm{ml}$ saliva was obtained from each participant between 7 and 8 am; and the samples were centrifuged at $13,053 \mathrm{x} \mathrm{g}$ for $\sim 10$ minutes to remove oral epithelial cells and food debris. The pure saliva samples were extracted and stored at $-20^{\circ} \mathrm{C}$ until required.

Ag colloids were synthesized using a deoxidizing method that was previously reported in the literature (30). Briefly, $4.5 \mathrm{ml}$ sodium hydroxide solution $(0.1 \mathrm{M}$; Yongda Chemical, Tianjin, China) was mixed with $5 \mathrm{ml}$ hydroxylamine hydrochloride solution $\left(6 \times 10^{-2} \mathrm{M}\right.$; Sinopharm Chemical, Shanghai, China), following which the mixture was added rapidly to $90 \mathrm{ml}$ silver nitrate aqueous solution $\left(1.11 \times 10^{-3} \mathrm{M}\right.$; Shanghai Reagent, Shanghai, China). The solution was stirred until it was uniformly gray. Finally, the Ag colloids were obtained by centrifuging the solution at $6,660 \mathrm{x}$ g for $10 \mathrm{~min}$ and discarding the supernatant. The absorption peak of Ag colloids was $\sim 418 \mathrm{~nm}$, with the full width at a half-maximum of $\sim 100 \mathrm{~nm}$. The Ag colloids had a diameter of $35 \pm 5 \mathrm{~nm}$ [mean \pm standard deviation (SD)].

SERS measurements. The saliva and Ag colloids were mixed in a ratio of $1: 1$, then a drop of the mixture was transferred onto an aluminum slide (Guantai Metal, Hebei, China) using a pipette and left to dry. The saliva SERS spectra were collected in the range of $400-1750 \mathrm{~cm}^{-1}$ using a confocal Raman micro-spectrometer (Renishaw, Wotton-under-Edge, Gloucestershire, UK). The parameters of the confocal Raman micro-spectrometer were set for a $785 \mathrm{~nm}$ diode laser, $2 \mathrm{~cm}^{-1}$ spectral resolution and Leica x20 objective lens (Leica Microsystems GmBH, Wetzlar, Germany) and an acquisition time of $10 \mathrm{sec}$. The SERS signal was detected with a Peltier cooled charge-coupled device camera (Renishaw, Wotton-under-Edge, UK). For each study participant, 1 saliva sample was analyzed with 3 repeated SERS measurements and the mean was recorded as the final SERS spectrum for subsequent data analysis.

Statistical analysis. Prior to statistical analysis, the raw spectra required preprocessing in order to remove experimental artifacts. The extraction of a pure Raman signal from the raw spectra involved the removal of the fluorescence background with a multi-polynomial fitting algorithm (31). All preprocessed SERS spectra were then normalized over an integrated area under the curve (AUC) at 400-1,750 $\mathrm{cm}^{-1}$ to allow an improved comparison of the spectral shapes and Raman band intensities between healthy volunteers and NPC patients. For PCA-LDA analysis, all normalized SERS spectral data sets were analyzed using SPSS software (SPSS, Inc., Chicago, IL, USA).

Often used in combination, PCA-LDA methods provide an efficient method for the data analysis of biological Raman spectroscopic datasets. PCA is a statistical technique for converting data from a higher dimensional space into a lower dimensional space, termed principal components (PCs) (18). Each PC was associated with the original saliva SERS spectrum by a variable termed the PC score, which represented the weight of the corresponding component. Once the PC scores 
Table I. NPC patient and healthy volunteer clinical information.

\begin{tabular}{lcc}
\hline Characteristic & $\begin{array}{c}\text { NPC } \\
\text { patients, } n\end{array}$ & $\begin{array}{c}\text { Healthy } \\
\text { volunteers, } \mathrm{n}\end{array}$ \\
\hline Total & 32 & 30 \\
Gender & & \\
$\quad$ Male & 23 & 23 \\
Female & 7 & 9 \\
Age, years & & \\
$\leq 50$ & 17 & 20 \\
$>50$ & 13 & 12 \\
Clinical stage of NPC & & \\
1 and 2 & 11 & NA \\
3 and 4 & 19 & NA \\
\hline
\end{tabular}

NPC, non-invasive nasopharyngeal carcinoma; NA, not applicable.

Table II. SERS peak positions and tentative vibrational mode assignments

\begin{tabular}{|c|c|c|}
\hline $\begin{array}{l}\text { Peak } \\
\text { position, } \mathrm{cm}^{-1}\end{array}$ & $\begin{array}{l}\text { Vibrational } \\
\text { mode }\end{array}$ & $\begin{array}{l}\text { Major } \\
\text { assignments }\end{array}$ \\
\hline 447 & Ring torsion & Phe \\
\hline 496 & 1 & Glycogen \\
\hline 590 & l & Ascorbic acid, Amide-VI \\
\hline 635 & $v(\mathrm{C}-\mathrm{S})$ & L-Tyr, Lactose \\
\hline 725 & $\delta(\mathrm{C}-\mathrm{H})$ & Adenine, $\mathrm{CoA}$ \\
\hline 812 & $v(\mathrm{C}-\mathrm{C}-\mathrm{O})$ & L-Ser \\
\hline 888 & $\delta(\mathrm{C}-\mathrm{O}-\mathrm{H})$ & D-GalN \\
\hline 1,003 & $v \delta(\mathrm{C}-\mathrm{C})$ & Phe \\
\hline 1,052 & $\mathrm{C}-\mathrm{O} / \mathrm{C}-\mathrm{N}$ stretching & Protein \\
\hline 1,134 & $v(\mathrm{C}-\mathrm{N})$ & D-Mannose \\
\hline 1,204 & Ring vibration & L-Tryp, Phe \\
\hline 1,270 & $\mathrm{C}=\mathrm{C}$ groups & UFA \\
\hline 1,336 & $v(\mathrm{C}-\mathrm{H})$ & Nucleic acid bases \\
\hline 1,448 & $\delta(\mathrm{CH} 2)$ & Collagen, PLP \\
\hline 1,619 & $v(\mathrm{C}=\mathrm{C})$ & Tryp \\
\hline 1,662 & 1 & Nucleic acid \\
\hline
\end{tabular}

$v$, stretching vibration; $\delta$, bending vibration; $v \delta$, symmetric stretch; Tryp, tryptophan; Phe, phenylalanine; Tyr, tyrosine; GalN, galactosamine; Ser, serine; UFA, unsaturated fatty acids; PLP, phospholipids; CoA, coenzyme A; /, indicates the vibrational mode is unclear.

for the saliva SERS spectra had been calculated, they were used for additional analysis instead of the original data. The most significant PCs were identified by independent-sample $t$-test, and were retained for the subsequent LDA analysis. $\mathrm{P}<0.05$ was considered to indicate a statistically significant difference. LDA maximized intergroup variance and minimized intragroup variance; therefore ensuring maximum separability between the NPC patients and the healthy volunteers. When combined with PCA, LDA was used to generate a diagnostic algorithm based on the most statistically significant PC scores with leave-one-out, cross-validation procedures. ROC curves were generated by successively changing the discrimination threshold levels to additionally evaluate the classifications of the PCA-LDA-based statistical techniques for NPC diagnosis.

\section{Results}

SERS spectra analysis. The saliva RS and saliva SERS spectra were produced using 1 saliva sample obtained from 1 NPC patient in order to evaluate the effects of the Ag colloid enhancement on the saliva RS. All spectra were measured under the same conditions with low excitation laser power $(5 \mathrm{~mW})$ and $10 \mathrm{sec}$ spectral data acquisition time, so as to avoid thermal denaturation of the biomolecular structure and degradation of the protein. The SERS spectrum with Ag colloid and the RS without Ag colloid obtained from the NPC patient are presented in Fig. 1A. It was observed that the intensity of the saliva SERS spectrum is enhanced compared with the intensity of the saliva RS. Few weak Raman peaks were present in the saliva RS without Ag colloid, due to limited detection sensitivity and strong autofluorescence background (Fig. 1A). When the $\mathrm{Ag}$ colloids were mixed with the saliva, the biomolecules of saliva were absorbed to the Ag colloid surfaces; therefore, the weak intensity of the numerous dominant vibration bands were enhanced significantly and the strong fluorescence background was decreased (Fig. 1A).

The normalized mean of the SERS spectra for the saliva samples of the 32 NPC patients and 30 healthy volunteers is presented in Fig. 1B. The SD of the mean is indicated by shading. The mean SERS spectrum of NPC patient saliva samples had an increased SD compared with the saliva SERS spectrum from the healthy volunteers, which implies that NPC patient saliva samples possess a greater number of constituent variations than the saliva of healthy volunteers. The saliva SERS spectrum of the NPC patients peaks at 447, 496, 590, 635, 729, 812, 888, 1,003, 1,052, 1,134, 1,204, $1,270,1,336,1,448,1,619$ and $1,662 \mathrm{~cm}^{-1}$. These peaks also appeared in the saliva SERS spectra of the healthy volunteers. The strongest peaks at 496, 635, 1,003 and $1,134 \mathrm{~cm}^{-1}$ existed in the patients and the volunteers. The difference in the SERS spectrum between NPC patients and the healthy volunteers (Fig. 1B) demonstrates the significant differences in SERS spectral intensities between the two groups. The intensity of the SERS peaks at 496, 635 and $1,134 \mathrm{~cm}^{-1}$ in NPC patient saliva were weaker than those in healthy volunteer saliva, while the NPC saliva SERS spectrum exhibited increased peaks at 447, 729, 1,003, 1,270 and $1,448 \mathrm{~cm}^{-1}$ compared with the healthy volunteers. The SERS peaks demonstrating the most significant differences between the two groups were located at 447, 496, 635, $729,1,134,1,270$ and $1,448 \mathrm{~cm}^{-1}$. Prominent SERS bands were tentatively assigned (Table II) to explore the molecular basis corresponding to the observed saliva SERS spectra $(10,18,32-37)$. The saliva SERS spectra of NPC patients was similar to that of healthy volunteers, but the key difference between the spectra were the intensity variations of the peaks indicating a biochemical variance during NPC formation. 
A

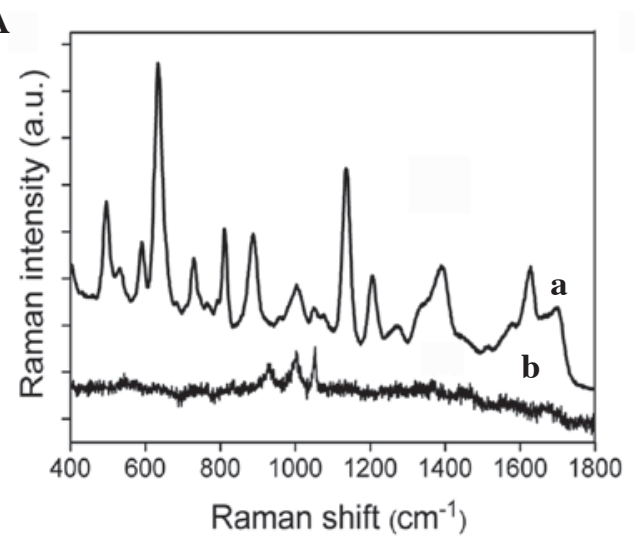

B

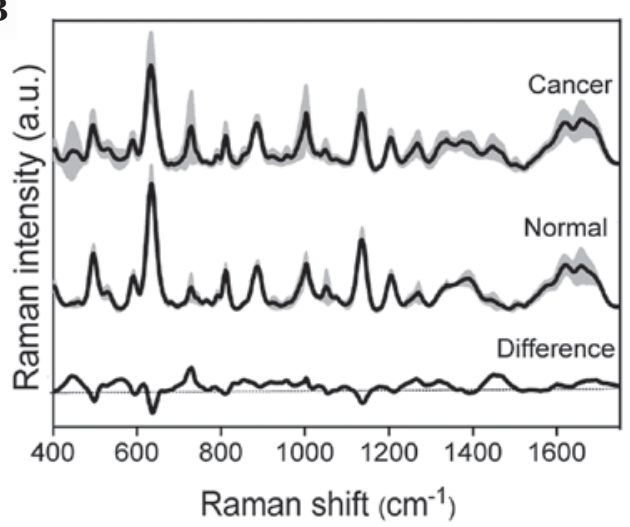

Figure 1. (Aa) SERS spectrum of the saliva of a NPC patient with Ag colloid. (b) Regular Raman spectrum of the NPC patient saliva without Ag colloid. (B) Comparison of the mean normalized saliva SERS spectra for NPC patients $(n=32)$ and the healthy volunteers ( $=30)$. The shaded regions demonstrate the standard deviation of the mean. The difference between SERS spectrums is revealed at the bottom of the graph. SERS, surface-enhanced Raman spectroscopy; NPC, nasopharyngeal carcinoma; Ag, silver.

A

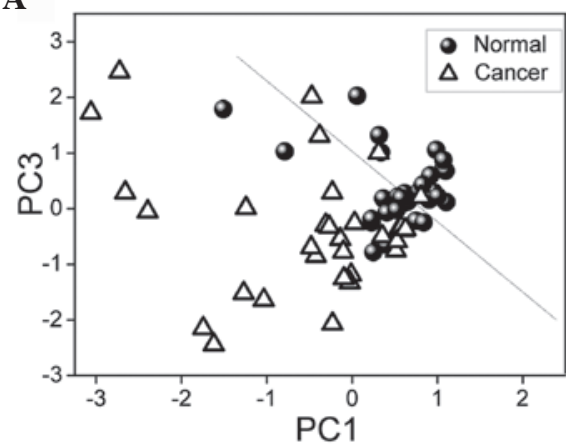

B

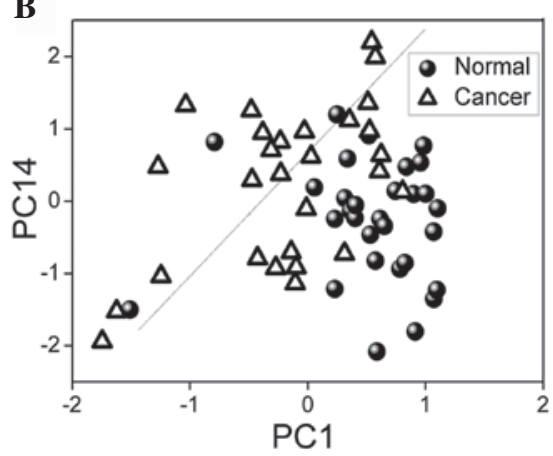

Figure 2. (A) Scatter plot of scores from 2 PCs (PC1, PC3) for healthy volunteers vs. NPC patients. The dotted line (PC3=-1.298PC1+1.013) distributed the two groups in 2 separate directions. (B) Scatter plot of scores from 2 PCs (PC1, PC14) for healthy volunteers vs. NPC patients. The dotted line (PC14=1.731PC1+0.653) distributed the two groups in 2 separate directions. PC, principal component; NPC, nasopharyngeal carcinoma.

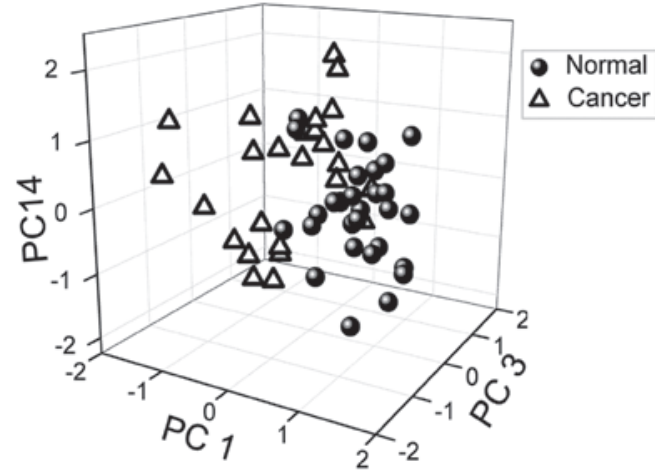

Figure 3. A three-dimensional scatter plot using PC1, PC3 and PC14 as the 3 axes for healthy volunteers (circles) vs. NPC patients (triangles). PC, principal component.

PCA-LDA analysis. PCA-LDA methods were used to analyze and differentiate saliva SERS spectra between NPC patients and the healthy volunteers in order to use the information gathered from the whole spectrum. The PC scores were calculated by PCA as variables for one independent-sample $t$-test. In total, 3 PCs (PC1, PC3 and PC14) were revealed to be the most diagnostically significant $(\mathrm{P}<0.05)$ for detecting NPC patients. Based on various combinations of significant
PCs (PC1 vs. PC3; PC1 vs. PC14), scatter plots were generated to compare NPC patients and healthy volunteers (Fig. 2). The NPC patient scores (triangles) and healthy volunteer scores (circles) were distributed in 2 separate directions, and the distribution of the healthy volunteer scores was narrower than that of the NPC patients for PC3 and PC14 (Fig. 2A and B). A three-dimensional scatter plot using PC1, PC 3 and PC14 as the 3 axes is presented in Fig 3. LDA with leave-one-out and cross-validation procedures was subsequently used on the three most significant PCs (PC1, PC3 and PC14) to generate diagnostic algorithms. A diagnostic sensitivity of 86.7 and $68.8 \%$, with a specificity of 80.0 and $68.8 \%$, could be achieved for the NPC patients and the healthy volunteers, respectively, when the separation lines, which classified NPC patients from healthy volunteers, were set in Fig. 2A-B. Posterior probability values of the healthy volunteers (circles) and NPC patients (triangles) were additionally calculated using a LDA model, as presented in Fig. 4A. When the discrimination threshold was set at 0.5 , the diagnostic sensitivity and specificity for distinguishing NPC patients from healthy volunteers was 86.7 and $86.7 \%$, respectively.

The diagnostic accuracy of the PCA-LDA-based diagnostic model for NPC detection was quantified by plotting a ROC curve (Fig. 4B). The ROC curve combined the diagnostic 

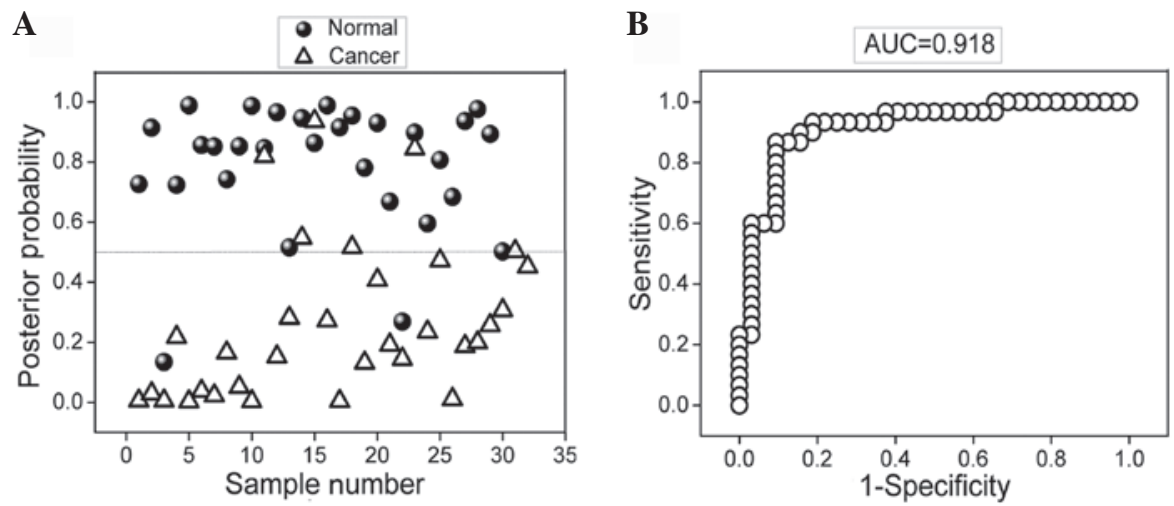

Figure 4. (A) Scatter plots of the posterior probability of the healthy volunteers (circles) and NPC patients (triangles) using a LDA model. The separation threshold was 0.5. (B) The ROC curve was used for quantifying the results of the diagnostic accuracy of a PCA-LDA based diagnostic model for NPC detection. The integrated AUC of the ROC curve was 0.918. ROC, receiver operating characteristic; AUC, area under the curve; PCA-LDA, principal component analysis and linear discriminant analysis.

sensitivity with the diagnostic specificity, which could indicate the association between sensitivity and specificity at various cutoff values of the probability. By changing the discrimination threshold in Fig. 4A, the diagnostic sensitivity may vary with the specificity in ROC curves. The AUC of the ROC curve was a measure of the diagnostic accuracy. An AUC of 1 represented a perfect test for discrimination between NPC patients and healthy volunteers, and an AUC of 0.5 indicated an ineffective test. Therefore, the closer the AUC was to 1, the greater the diagnostic capacity. By plotting the sensitivity against specificity, the present study demonstrated an AUC of 0.918 , which was associated with an overall diagnostic accuracy of $83.9 \%$, sensitivity of $86.7 \%$ and specificity of $81.3 \%$. These results confirmed the outstanding diagnostic accuracy of the PCA-LDA-based diagnostic algorithm for NPC detection.

\section{Discussion}

The present study demonstrated that specific biomolecular differences between the saliva of NPC patients and the saliva of healthy volunteers could be detected using SERS spectra. Furthermore, saliva SERS analysis combined with PCA-LDA diagnostic algorithms could achieve a high diagnostic accuracy, indicated by a sensitivity of $86.7 \%$, specificity of $81.3 \%$ and accuracy of $83.9 \%$, demonstrating the potential for the application of saliva SERS in NPC diagnosis. Numerous biomolecules, including proteins, lipids and nucleic acids, may be altered in quantity or confirmation, due to the alterations in metabolic processes during NPC formation. The molecular vibrations specific to certain types of these biomolecules may be reflected in SERS spectra, as reported in Table II. The SD of the mean for saliva SERS spectrum of the NPC patients was larger than that of healthy volunteers (Fig. 1B), possibly due to the alterations in saliva constituents in each patient as a result of the various disease stages of patients. In addition, a similar spectrum existed between the saliva of NPC patients and healthy volunteers; the major differences were the intensity of the peaks, indicating that the quantity of biomolecules varied with NPC formation. In the present study, the SERS peak centered at $729 \mathrm{~cm}^{-1}$, which is the C-H in-plane bending mode of adenine, demonstrates a higher intensity in the NPC patients, indicating that the amount of DNA or RNA in the saliva of NPC patients was increased. There were other peaks in the spectra presented in Fig. 1B, at 1336 and $1662 \mathrm{~cm}^{-1}$, that indicated nucleic acid presence. The current study revealed that circulating DNA mainly resulted from apoptosis of carcinoma cells and necrosis in the tumor microenvironment. The secretion of tumor tissue, necrosis and apoptosis of tumor cells results in the debris being engulfed by macrophages, which releases digested nucleic acids (38). Furthermore, NPC is located near to the oral cavity, where metabolites, including DNA, RNA or proteins, may be drained to the saliva. Feng et al reported results similar to the findings demonstrated in the present study $(18,39)$.

Collagen is the main component of all types of human tissue and is important in the formation and stability of the extracellular matrix and basal membrane (ECM-BM). Previous studies confirmed that the structural integrity of the ECM-BM was closely associated with carcinoma formation, development, invasion and prognosis of the patient $(40,41)$. In various carcinomas, the BM usually becomes thin and easily degrades, releasing collagen into the surrounding environment. Therefore, in the present study, the SERS band at $1,448 \mathrm{~cm}^{-1}$, which originates from the $\mathrm{CH}_{2}$ bending mode of collagen, demonstrated an increased signal in NPC patient saliva compared with the saliva of healthy volunteers. This peak was also observed in other studies $(18,39)$.

Phenylalanine demonstrated a strong SERS peak of $1,003 \mathrm{~cm}^{-1}$, which is assigned to a C-C symmetric stretch. The $1,003 \mathrm{~cm}^{-1}$ SERS signal was more intense in the saliva of NPC patients, suggesting that NPC patient saliva possesses a relatively higher content of phenylalanine compared with healthy volunteers. This finding has also been confirmed in lung carcinoma tissue (10). The tumor cell proliferation process consumes energy, and in the majority of tumor cells there is a rise in glycometabolism, which maintains survival, growth, invasion and metastasis of the tumor cells, leading to a lower glycogen threshold. In the current study, the SERS signal for glycogen was $\sim 1,134 \mathrm{~cm}^{-1}$ and D-mannose was $496 \mathrm{~cm}^{-1}$. These were decreased in NPC patient saliva compared to healthy volunteer saliva, demonstrating that glycometabolism in NPC patients was more vigorous. Tumor cells demonstrate extremely high endogenous fatty acid synthesis, regardless 
of the level of circulating fatty acids. Circulating fatty acids may directly promote tumor cell growth and metastasis (42). The characteristic SERS peak attributed to the C-C waver mode of fatty acids was $1,270 \mathrm{~cm}^{-1}$ in the present study. The present study findings demonstrated that the NPC patient saliva was associated with an increased level of fatty acids. In addition, as compared to the healthy volunteers, the saliva SERS bands of the NPC patients revealed lower intensities at $635 \mathrm{~cm}^{-1}$ (tyrosine) and $812 \mathrm{~cm}^{-1}$ (L-serine), and a higher intensity at $1619 \mathrm{~cm}^{-1}$ (tryptophan); therefore, demonstrating an increase or decrease in the levels of certain amino acids. The distinctive difference in SERS spectra between NPC patients and healthy volunteers reinforced that saliva SERS could be used to reveal molecular alterations associated with NPC formation.

Carcinoma formation is a long-term, multi-stage, dynamic process. During tumor development, minor alterations in tumor cells may bring about a corresponding variation in metabolites. Therefore, using metabolomics may lead to a higher diagnostic sensitivity in the early diagnosis of carcinoma (43). The metabolic spectrum contains information concerning all metabolites during carcinoma formation. In the present study, SERS spectra analysis only used certain regions of the spectrum; therefore, multivariate statistical analysis (PCA-LDA) was used, which incorporated the entire Raman spectra data for analysis. In the current study, Figs. 2 and 3 demonstrated that the PC scores of the NPC patients and healthy volunteers were distributed in 2 separate directions, revealing that the NPC patients could be easily differentiated from the healthy volunteers. The PCA-LDA modeling provided a diagnostic sensitivity of $86.7 \%$ and a specificity of $81.3 \%$. The ROC curve (Fig. 4B) of PCA-LDA modeling (AUC=0.918) was associated with an overall diagnostic accuracy of $83.9 \%$, which verified that the saliva SERS spectra integrated with a PCA-LDA algorithm provides a good diagnostic efficacy. Overall, these results confirmed that saliva SERS spectra could be used for early NPC detection, due to the excellent diagnostic performance.

In summary, the present study revealed significant differences in saliva SERS spectra between NPC patients and healthy volunteers, and tentative assignments of the prominent SERS bands were associated with proteins, nucleic acids, fatty acids, glycogen and collagen. Furthermore, the PCA-LDA methods employed to classify SERS spectra for NPC identification demonstrated a good diagnostic sensitivity of $86.7 \%$, specificity of $81.3 \%$ and accuracy of $83.9 \%$. These findings identify that saliva SERS used in conjunction with a PCA-LDA algorithm is feasible for the non-invasive detection of NPC. Additional studies may continue to evaluate saliva SERS data, in order to confirm the utility of this novel, convenient carcinoma diagnostic method and may investigate this method for tumor stage detection.

\section{Acknowledgements}

The present study was funded by the National Clinical Key Specialty Construction Program (grant no., 61210016), Key Clinical Specialty Discipline Construction Program of Fujian (grant no., 61178090), People's Republic of China and Fujian Provincial Key Laboratory of Translational Cancer Medicine (grant no., 61178083), the Provincial Natural Science Foundation (grant no. 2015J01436), National Natural Science Foundation of China (grant no., 61405036), Science and Technology Project of Fujian Province (grant no., WKJ-FJ-01), Fujian Province Health Commission Young and Middle-aged Talent Training Project (grant no., 2014-ZQN-JC-6) and Program for Chang Jiang Scholars and Innovative Research Team in University (grant no., IRT1115).

\section{References}

1. Wei WI and Sham JS: Nasopharyngeal carcinoma. Lancet 365: 2041-2054, 2005

2. Su SF, Han F, Zhao C, Chen CY, Xiao WW, Li JX and Lu TX: Long-term outcomes of early-stage nasopharyngeal carcinoma patients treated with intensity-modulated radiotherapy alone. Int J Radiat Oncol Biol Phys 82: 327-333, 2012.

3. Ma J, Mai HQ, Hong MH, Min HQ, Mao ZD, Cui NJ, Lu TX and Mo HY: Results of a prospective randomized trial comparing neoadjuvant chemotherapy plus radiotherapy with radiotherapy alone in patients with locoregionally advanced nasopharyngeal carcinoma. J Clin Oncol 19: 1350-1357, 2001.

4. Guigay J: Advances in nasopharyngeal carcinoma. Curr Opin Oncol 20: 264-269, 2008.

5. King AD, Ma BB, Yau YY, Zee B, Leung SF, Wong JKT, Kam MKM, Ahuja AT and Chan ATC: The impact of 18F-FDG $\mathrm{PET} / \mathrm{CT}$ on assessment of nasopharyngeal cancer at diagnosis. $\mathrm{Br}$ J Radiol 81: 291-298, 2008.

6. Lee AW, Lin JC and Ng WT: Current management of nasopharyngeal cancer. Semin Radiat Oncol 22: 233-244, 2012.

7. Chan KC, Hung EC, Woo JK, Chan PK, Leung SF, Lai FP, Cheng AS, Yeung SW, Chan YW, Tsui TK, et al: Early detection of nasopharyngeal carcinoma by plasma EpsteinBarr virus DNA analysis in a surveillance program. Cancer 119: 1838-1844, 2013.

8. Lee SY, Rhee CM, Leung AM, Braverman LE, Brent GA and Pearce EN: A review: Radiographic iodinated contrast media-induced thyroid dysfunction. J Clin Endocrinol Metab 100: 376-83, 2015.

9. Gonchukov SA, Lonkina TV, Minaeva SA, Sundukov AV, Migmanov TE, Lademann J, Darvin ME and Bagratashvili VN: Confocal Raman microscopy of pathologic cells in cerebrospinal fluid. Laser Phys Lett 11: 015602, 2014.

10. Huang Z, McWilliams A, Lui H, McLean DI, Lam S and Zeng H: Near-infrared Raman spectroscopy for optical diagnosis of lung cancer. Int J Cancer 107: 1047-1052, 2003.

11. Lui H, Zhao J, McLean D and Zeng H: Real-time Raman spectroscopy for in vivo skin cancer diagnosis. Cancer Res 72: 2491-2500, 2012.

12. Huang Z, McWilliams A, Lam S, English J, McLean DI, Lui H and Zeng $\mathrm{H}$ : Effect of formalin fixation on the near-infrared Raman spectroscopy of normal and cancerous human bronchial tissues. Int J Oncol 23: 649-655, 2003.

13. Widjaja E, Zheng W and Huang Z: Classification of colonic tissues using near-infrared Raman spectroscopy and support vector machines. Int J Oncol 32: 653-662, 2008.

14. Lademann J, Caspers PJ, van der Pol A, Richter H, Patzeit A, Zastrow L, Darvin M, Sterry W and Fluhr JW: In vivo Raman spectroscopy detects increased epidermal antioxidative potential with topically applied carotenoids. Laser Phys Lett 6: 76-79, 2009.

15. Werncke W, Latka I, Sassning S, Dietzek B, Darvin ME, Meinke MC, Popp J, König K, Fluhr JW and Lademann J: Two-color Raman spectroscopy for the simultaneous detection of chemotherapeutics and antioxidative status of human skin. Laser Phys Lett 8: 895, 2011.

16. Li SX, Zhang YJ, Zeng QY, Li LF, Guo ZY, Liu ZM, Xiong HL and Liu SH: Potential of cancer screening with serum surface-enhanced Raman spectroscopy and a support vector machine. Laser Phys Lett 11: 065603, 2014.

17. Johansson P, Xu H and Käll M: Surface-enhanced Raman scattering and fluorescence near metal nanoparticles. Physical Review B 72: 035427, 2005.

18. Feng S, Chen R, Lin J, Pan J, Chen G, Li Y, Cheng M, Huang Z, Chen J and Zeng H: Nasopharyngeal cancer detection based on blood plasma surface-enhanced Raman spectroscopy and multivariate analysis. Biosens Bioelectron 25: 2414-2419, 2010. 
19. Bell SE, Mackle JN and Sirimuthu NM: Quantitative surface-enhanced Raman spectroscopy of dipicolinic acid - towards rapid anthrax endospore detection. Analyst 130: 545-549, 2005.

20. Lin J, Yu Y, Li B, Huang H, Lin S, Li C, Su Y, Feng S, Chen G, Li Y, et al: Electrical pulse - mediated enhanced delivery of silver nanoparticles into living suspension cells for surface enhanced Raman spectroscopy. Laser Phys Lett 9: 240-246, 2012.

21. Harper MM, Dougan JA, Shand NC, Graham D and Faulds K: Detection of SERS active labelled DNA based on surface affinity to silver nanoparticles. Analyst 137: 2063-2068, 2012.

22. Huang H, Lin D, Chen W, Yu Y, Xu J, Liang Z, Lin X, Dong Z and Shi $\mathrm{H}$ : Nondestructive discrimination between normal and hematological malignancy cell lines using near-infrared Raman spectroscopy and multivariate analysis. Laser Phys Lett 11: 085601, 2014

23. Li Z, Li C, Lin D, Huang Z, Pan J, Chen G, Lin J, Liu N, Yu Y, Feng $S$ and Chen R: Surface-enhanced Raman spectroscopy for differentiation between benign and malignant thyroid tissues Laser Phys Lett 11: 045602, 2014.

24. Wang X, Qian X, Beitler JJ, Chen ZG, Khuri FR, Lewis MM, Shin HJ, Nie S and Shin DM: Detection of circulating tumor cells in human peripheral blood using surface-enhanced Raman scattering nanoparticles. Cancer Res 71: 1526-1532, 2011.

25. Feng S, Lin J, Cheng M, Li YZ, Chen G, Huang Z, Yu Y, Chen R and Zeng H: Gold nanoparticle based surface-enhanced Raman scattering spectroscopy of cancerous and normal nasopharyngeal tissues under near-infrared laser excitation. Appl Spectrosc 63: 1089-1094, 2009.

26. Gao K, Zhou H, Zhang L, Lee JW, Zhou Q, Hu S, Wolinsky LE, Farrell J, Eibl G and Wong DT: Systemic disease-induced salivary biomarker profiles in mouse models of melanoma and non-small cell lung cancer. PLoS One 4: e5875, 2009.

27. Kho KW, Malini O, Shen ZX and Soo KC: Surface enhanced Raman spectroscopic (SERS) study of saliva in the early detection of oral cancer. Proceedings of SPIE 5702: 84-91, 2005.

28. Li X, Yang T and Lin J: Spectral analysis of human saliva for detection of lung cancer using surface-enhanced Raman spectroscopy. J Biomed Opt 17: 037003, 2012.

29. Feng S, Lin D, Lin J, Huang Z, Chen G and Li Y: Saliva analysis combining membrane protein purification with surface-enhanced Raman spectroscopy for nasopharyngeal cancer detection. Appl Phys Lett 104: 073702, 2014.

30. Leopold N and Lendl B: A new method for fast preparation of highly surface-enhanced Raman scattering (SERS) active silver colloids at room temperature by reduction of silver nitrate with hydroxylamine hydrochloride. J Phys Chem B 107: 5723-5727, 2003.
31. Zhao J, Lui H, McLean DI and Zeng H: Automated autofluorescence background subtraction algorithm for biomedical Raman spectroscopy. Appl Spectrosc 61: 1225-1232, 2007.

32. Mahadevan-Jansen A and Richards-Kortum R: Raman spectroscopy for cancer detection: A review. In: Proceedings of the 19th Annual International Conference of the IEEE. Chicago, IL, USA 6: 2722-2728, 1997.

33. Krafft C, Neudert L, Simat T and Salzer R: Near infrared Raman spectra of human brain lipids. Spectrochim Acta A Mol Biomol Spectrosc 61: 1529-1535, 2005.

34. Stone N, Kendall C, Shepherd N, Crow P and Barr H: Near-infrared Raman spectroscopy for the classification of epithelial pre-cancers and cancers. J Raman Spectrosc 33: 564-573, 2002.

35. Schulz $\mathrm{H}$ and Baranska M: Identification and quantification of valuable plant substances by IR and Raman spectroscopy. Vibrational Spectrose 43: 13-25, 2007.

36. Movasaghi Z, Rehman S and Rehman IU: Raman spectroscopy of biological tissues. Appl Spectrosc Rev 42: 493-541, 2007.

37. Feng S, Lin D, Lin J, Li B, Huang Z, Chen G, Zhang W, Wang L, Pan J, Chen R and Zeng H: Blood plasma surface-enhanced Raman spectroscopy for non-invasive optical detection of cervical cancer. Analyst 138: 3967-3974, 2013.

38. Gahan PB, Anker P and Stroun M: Metabolic DNA as the origin of spontaneously released DNA? Ann N Y Acad Sci 1137: 7-17, 2008.

39. Feng S, Chen R, Lin J, Pan J, Wu Y, Li Y, Chen J and Zeng H: Gastric cancer detection based on blood plasma surface-enhanced Raman spectroscopy excited by polarized laser light. Biosens Bioelectron 26: 3167-3174, 2011.

40. Kannan S, Balaram P, Chandran G, Pillai MR, Mathew B, Nalinakumari KR and Nair MK: Alterations in expression of basement membrane proteins during tumour progression in oral mucosa. Histopathology 24: 531-537, 1994.

41. Firth NA and Reade PC: The prognosis of oral mucosal squamous cell carcinomas: A comparison of clinical and histopathological grading and of laminin and type IV collagen staining. Aust Dent J 41: 83-86, 1996.

42. Bauer DE, Hatzivassiliou G, Zhao F, Andreadis C and Thompson CB: ATP citrate lyase is an important component of cell growth and transformation. Oncogene 24: 6314-6322, 2005.

43. Schmidt C: Metabolomics takes its place as latest up-and-coming "omic" science. J Natl Cancer Inst 96: 732-734, 2004. 